\title{
Profile of differentially expressed miRNAs in high-grade serous carcinoma and clear cell ovarian carcinoma, and the expression of miR-510 in ovarian carcinoma
}

\author{
XINCHEN ZHANG $^{1 *}$, GORDON GUO $^{2 *}$, GUANG WANG $^{1}$, JINYAO ZHAO $^{1}$, \\ $\mathrm{BO} \mathrm{WANG}^{3}$, XIAOTANG YU ${ }^{3 * *}$ and YANFANG DING ${ }^{1 * *}$ \\ ${ }^{1}$ Department of Histology and Embryology, Dalian Medical University, Dalian, Liaoning 116044, P.R. China; \\ ${ }^{2}$ Department of Radiation Oncology, University of Manitoba, Winnipeg, Manitoba R3T 2N2, Canada; \\ ${ }^{3}$ Department of Pathology and Forensic Medicine, Dalian Medical University, Dalian, Liaoning 116044, P.R. China
}

Received November 18,2014; Accepted August 27, 2015

DOI: $10.3892 / \mathrm{mmr} .2015 .4485$

\begin{abstract}
Improved insight into the molecular and genetic profile of different types of epithelial ovarian cancer (EOC) is required for understanding the carcinogenesis of EOC and may potentially be exploited by future targeted therapies. The aim of the present study was to identify a unique microRNA (miRNA) patterns and key miRNAs, which may assist in predicting progression and prognosis in high-grade serous carcinoma (HGSC) and clear cell carcinoma (CCC). To identify unique miRNA patterns associated with HGSC and CCC, a miRNA microarray was performed using Chinese tumor bank specimens of patients with HGSC or CCC in a retrospective analysis. The expression levels of four deregulated miRNAs were further validated using reverse transcription-quantitative polymerase chain reaction (RT-qPCR) in an external cohort of 42 cases of HGSC and 36 cases of CCC. Kaplan-Meier analysis was performed to analyze the correlation between the expression levels of the four miRNAs and patient prognosis. Among these validated miRNAs, miR-510 was further examined in another cohort of normal ovarian tissues, as well as the HGSC, low-grade serous carcinoma (LGSC) and CCC specimens using RT-qPCR and in situ hybridization.
\end{abstract}

Correspondence to: Miss. Yanfang Ding, Department of Histology and Embryology, Dalian Medical University, 9 LvShun South Road, Dalian, Liaoning 116044, P.R. China

E-mail: dingyanfang1201@yahoo.ca

Miss. Xiaotang Yu, Department of Pathology and Forensic Medicine, Dalian Medical University, 9 LvShun South Road, Dalian, Liaoning 116044, P.R. China

E-mail: leahyxt@163.com

\section{*,** Contributed equally}

Key words: ovarian carcinoma, high-grade serous carcinoma, clear cell carcinoma, low-grade serous carcinoma, microRNA, Hsa-miR-510
The results revealed that, of the 768 miRNAs analyzed in the microarray, 33 and 50 miRNAs were significantly upregulated and downregulated, respectively, with at least a 2-fold difference in HGSC, compared with CCC. The quantitative analysis demonstrated that miR-510 and miR-129-3p were significantly downregulated, and that miR-483-5p and miR-miR-449a were significantly upregulated in CCC, compared with HGSC $(\mathrm{P}<0.05)$, which was consistent with the microarray results. Kaplan-Meier analysis revealed low expression levels of miR-510 and low expression levels of miR-129-3p, advanced International Federation of Gynecology and Obstetrics (FIGO) stage, lymphatic metastasis and that HGSC was significantly associated with the poorer overall survival rates $(\mathrm{P}<0.05)$. The expression of miR-510 was significantly higher in the LGSC and CCC tissues, compared with the HGSC and normal ovarian tissues. The results of the present study suggested that different subtypes of EOC have specific miRNA signatures, and that miR-510 may be involved differently in HGSC and CCC. Thus, miR-510 and miR-129-3p may be considered as potential novel candidate clinical biomarkers for predicting the outcome of EOC.

\section{Introduction}

Ovarian cancer accounts for approximately one quarter of gynecological malignancies, however, it is the most life-threatening (1). Epithelial ovarian cancer (EOC) is the most common type of ovarian cancer, accounting for $90 \%$ of cases (2). Despite advances in surgery and chemotherapy, the prognosis remains poor, with a five-year-survival rate of $<45 \%$ worldwide $(3,4)$. The extent of disease, which is expressed as the International Federation of Gynecology and Obstetrics (FIGO) stage, success of primary surgery and histopathological features of the tumor are important prognostic markers $(5,6)$.

Based on investigations combining morphological features and immunohistochemistry, EOC can be broadly subdivided into high-grade serous carcinoma (HGSC), low-grade serous carcinoma (LGSC), clear cell carcinoma (CCC), mucinous carcinoma (MC) and endometrioid carcinoma (EC) (6). Different subtypes of EOC are associated with variable clinical 
manifestations, clinical outcomes, prognoses, sensitivity to chemotherapy, and associated with different underlying molecular abnormalities (7).

HGSC is the most common type of ovarian carcinoma, representing $80-85 \%$ of all cases of EOC in the West, and are well represented among the types of carcinomas, which present at an advanced FIGO stage (III or IV) (8). CCC comprises $\sim 5 \%$ of all ovarian tumors in North America, whereas they account for a larger proportion of ovarian tumors in Japan and China (8). They are most often at an early stage at presentation, and account for $>25 \%$ of all FIGO stage I and II EOCs $(6,9)$. Their distinct morphological features also correspond to unique underlying molecular abnormalities and genetic profiles (10). Investigating the molecular and genetic profiles of different types of EOC may assist in improving current understanding of the carcinogenesis of EOC and these may potentially be exploited by future targeted therapies.

MicroRNAs (miRNAs) are 19-25 nucleotide-long, noncoding RNAs, which regulate gene expression by repressing mRNA translation and/or directing mRNA cleavage. It has been reported that miRNAs are aberrantly expressed or mutated in cancer, suggesting that they may be involved in the initiation and progression of cancer (11). miRNAs are important as a novel class of oncogenes or tumor suppressor genes, depending on the targets they regulate (12). A number of studies have reported that miRNA expression signatures are associated with specific tumor subtypes, clinical outcomes, stages and responses to therapy $(11,13)$. Various miRNA gene expression analytical approaches, including microarrays and reverse transcription-quantitative polymerase chain reaction (RT-qPCR), have identified aberrantly expressed miRNAs in EOC, of which a number are associated with progression, classification, FIGO stage, prognosis, chemotherapy resistance (14-16). However, there are few studies concerning the difference in miRNA expression profile between HGSC and $\mathrm{CCC}$.

The aim of the present study was to identify miRNAs that were differentially expressed between subtypes of EOC, predominantly HGSC and CCC. The results identified several important miRNAs that were differentially expressed between HGSC and CCC, including miR-510. The possible clinical significance and prognostic value of these dysregulated miRNAs were subsequently investigated. The potential significance of miR-510 in EOC was further examined in another cohort of normal ovarian tissue samples, HGSC, LGSC and CCC specimens, using RT-qPCR and in situ hybridization (ISH). Identification of these miRNAs and further examination of their function role could lead to the identification of novel targets and/or biomarkers that could benefit patients with ovarian cancer.

\section{Patients and methods}

Patient samples. Patients who were diagnosed with EOC between 2004 and 2011 at the Obstetrics and Gynecology Hospital of Dalian (Liaoning, China), according to a pathological report, were recruited for the present study, which was approved by the Institutional Review Board of the Ministry of Science and Technology of China, the Human Resource Management Office (Beijing, China) and the ethics committee of the Dalian Medical University (Dalian, China). All participants signed a consent form prior to the surgical procedure and the investigations. Pathological specimens $\left(10 \times 10 \times 3 \mathrm{~mm}^{3}\right)$, which were collected from primary surgery were routinely fixed in formalin (Kangnaixin Biology Co., Zhongshan, China) and embedded in paraffin (Hongming Chemical Reagent Co., Jining, China). Each slide was re-evaluated by an expert pathologist in a blinded-manner, prior to the experiments being performed. The cases were classified according to the FIGO staging system (17). Only specimens containing $>70 \%$ tumor tissue were used for subsequent experiments. Clinicopathological data were also collected, including subtypes, age, FIGO stage and status of lymphatic metastasis. The histological classification and clinical staging were performed according to the World Health Organization classification (5) and FIGO staging (17), respectively.

The tumor samples comprised primary ovarian cancer obtained from surgery prior to chemotherapy. The clinicopathological features are presented in Table I. For miRNA microarray analysis, formalin-fixed, paraffin-embedded (FFPE) samples of EOC, comprising 20 cases of HGSC and 16 cases of CCC were collected. For validation, a separate cohort of patients, with complete prognosis data were selected, The FFPE specimens of HGSC $(n=22)$ and CCC $(n=20)$ were used in RT-qPCR. RT-qPCR was also used for the samples included in the microarray. For the investigation of miR-510 in normal ovarian epithelium and EOC, 10 samples of normal ovarian epithelium and 10 samples of LGSC tissue were included.

RNA extraction. Total RNA was extracted from the FFPE tissue samples from the patients with ovarian serous carcinoma (OSC) and CCC using an Ambion mirVana microRNA isolation kit (Ambion Life Technologies, Austin, TX, USA), according to the manufacturer's instructions. Briefly, FFPE tissue sections of $100-\mu \mathrm{m}$ thickness were deparaffinized with xylene (Liaoning Quan Rui Reagent Co. Ltd., Liaoning, China) at $50^{\circ} \mathrm{C}$, the specimens were washed in ethanol and digested with $10 \%$ proteinase K (Amresco Inc., Solon, OH, USA) at $55^{\circ} \mathrm{C}$ for $1-3 \mathrm{~h}$, depending on the tissue properties. RNA was extracted with acid phenol:chloroform (Ambion Life Technologies), followed by ethanol precipitation and DNAse (Takara Biotechnology Co., Ltd., Dalian, China) digestion. The quantity and quality of the total RNA was verified using a NanoVue spectrophotometer (GE Healthcare Life Sciences, Amersham, UK) and a Bioanalyzer 2100 (Agilent Technologies, Inc., Santa Clara, CA, USA), according to the manufacturer's instructions. All samples exhibited adequate RNA quantity and quality.

miRNA microarray and data analysis. The miRNA microarray was performed at the Shannon McCormack Advanced Molecular Diagnostics Laboratory Research Services, Dana Farber Cancer Institute, Harvard Clinic and Translational Science Center (Boston, MA, USA). A microarray platform, optimized for the analysis of a panel of 768 human miRNAs (TaqMan ${ }^{\circledR}$ Array Human MicroRNA Card Set v2.0; Thermo Fisher Scientific Inc., Waltham, MA, USA) was used to analyze and compare the patterns of miRNA expression in the 20 cases of HGSC and 16 cases of CCC. Individual RT-qPCR assays 
Table I. Clinicopathological information for patients selected for microarray and RT-qPCR analyses.

\begin{tabular}{|c|c|c|c|}
\hline \multirow[b]{2}{*}{ Parameter } & \multicolumn{2}{|c|}{ OSC } & \multirow[b]{2}{*}{$\mathrm{CCC}$} \\
\hline & HGSC & LGSC & \\
\hline Microarray & 20 & & 16 \\
\hline Age (mean \pm SEM) & $53.6 \pm 7.1$ & & $47.3 \pm 8.3$ \\
\hline \multicolumn{4}{|l|}{ FIGO stage } \\
\hline I & & 16 & \\
\hline II & 1 & & \\
\hline III & 17 & & \\
\hline IV & 2 & & \\
\hline Validation cohort (RT-qPCR) & 42 & 10 & 36 \\
\hline Age (mean \pm SEM) & $52.8 \pm 10.4$ & $45.9 \pm 8.1$ & $46.4 \pm 9.1$ \\
\hline \multicolumn{4}{|l|}{ FIGO stage } \\
\hline $\mathrm{I}$ & 2 & 8 & 31 \\
\hline II & 3 & 2 & 1 \\
\hline III & 35 & & 4 \\
\hline IV & 2 & & \\
\hline \multicolumn{4}{|c|}{ Chemosensitivity (only available for HGSC) } \\
\hline $\mathrm{CR}$ & 26 & & \\
\hline IR & 16 & & \\
\hline \multicolumn{4}{|l|}{ Status of follow up } \\
\hline Alive & 18 & 10 & 31 \\
\hline Succumbed to mortality & 24 & 0 & 5 \\
\hline
\end{tabular}

OSC, ovarian serous carcinoma; HGSC, high-grade serous ovarian carcinoma; CCC, clear cell ovarian carcinoma; CR, complete response; IR, incomplete response; RT-qPCR, reverse transcription-quantitative polymerase chain reaction, SEM, standard error of the mean.

were formatted into a TaqMan low-density array (Applied Biosystems Life Technologies, Foster City, CA, USA). The normalized microarray data were managed and analyzed using Statminer version 3.0 (Integromics ${ }^{\mathrm{TM}}$, Granada, Spain).

$R T-q P C R$. The miRNA expression levels were determined using RT-qPCR with commercial primers of the GenePharma miRNA-specific RT primer and miRNA-specific PCR primer set (forward and reverse; Shanghai GenePharma Co., Ltd., Shanghai, China). The following primers were used: miR-483-5p sense, CAGATCAATAAGACGGGAGGAA, and antisense, TATGCTTGTTCTCGTCTCTGTGTC; miR-510 sense, CTTCCATACTCAGGAGAGTGGC and antisense, TATCGTTGTACTCCAGACCAAGAC; miR-129-3p sense, CGCGAATCTTTTTGCGGTCT, and antisense, CCGCAAATGCTTTTTGGGGT; miR-449a sense, GTGTGATGAGCTGGCAGTGTA, and antisense, AGCAGTTGCATGTTAGCCGAT. Briefly, specific miRNAs were generated from 220-300 ng of total RNA in a single-step reaction using an RT kit (cat. no. DRR037A; Takara Biotechnology Co.), according to the manufacturer's instructions. PCR amplification was performed using the specific commercial primers (Shanghai GenePharma Co., Ltd.) with $1 \mu 1$ of the RT production/well. The reactions were performed in a 96-well optical plate at $95^{\circ} \mathrm{C}$ for $3 \mathrm{~min}$, followed by 40 cycles of $95^{\circ} \mathrm{C}$ for $12 \mathrm{sec}$ and $62^{\circ} \mathrm{C}$ for $40 \mathrm{sec}$, using U6 as a housekeeping gene.
The experiments were run in triplicate for each case, to allow for technical variability. qPCR was performed on a Stratagern Mx3000P (Agilent Technologies, Inc.). The data were analyzed using Mx3000P software. The relative microRNA expression levels were calculated using the $2^{-\Delta \Delta \mathrm{Cq}}$ method (18).

ISH of miR-510. ISH was performed on the FFPE sections, according to previously described methods (19). A commercially available probe for the miR-510 (Exiqon, Inc., Woburn, MA, USA) was used, and procedures were performed according to standard protocols. Briefly, 3-5 $\mu \mathrm{m}$ sections of tissues were deparaffinized and dehydrated, followed by incubation in $20 \%$ sodium bisulphate $/ 2 \mathrm{X}$ standard saline citrate (SSC; Thermo Fisher Scientific, Inc.) at $75^{\circ} \mathrm{C}$ for $20 \mathrm{~min}$. Following washing in $2 \mathrm{X} \mathrm{SSC}$, the slides were treated with proteinase $\mathrm{K}(30 \mu \mathrm{g} / \mathrm{ml}$, Amresco Inc.; cat. no. 1227B016) for $5 \mathrm{~min}$, followed by three washes in phosphate-buffered saline. The sections were incubated with pre-hybridization buffer [10 ml formamide (Amresco Inc.), $5 \mathrm{ml}$ 20X SSC, $2 \mathrm{ml} 50 \mathrm{X}$ Denhardt's solution, $250 \mu \mathrm{l} 20 \mathrm{mg} / \mathrm{ml}$ yeast RNA, 1,000 $\mu \mathrm{l}$ $10 \mathrm{mg} / \mathrm{ml}$ salmon sperm DNA (Life Technologies, Carlsbad, CA, USA), $0.4 \mathrm{~g}$ blocking powder (Roche, Basel, Switzerland) and $1.75 \mathrm{ml}$ diethylpyrocarbonate-treated water (Amresco Inc.)] at $55^{\circ} \mathrm{C}$ for $1 \mathrm{~h}$. Hybridization buffer containing the probes for has-miR-510 was added to each section and hybridized overnight at $55^{\circ} \mathrm{C}$. Following hybridization and washing with 
2X SSC, the sections were incubated with anti-DIG-Fab-AP (cat. no. 11376621; Roche Diagnostics GmbH, Mannheim, Germany) for $2 \mathrm{~h}$ at room temperature. Following three washes with NT buffer (Thermo Fisher Scientific, Inc.), the sections were stained with BCIP $(3.5 \mu \mathrm{l} / \mathrm{ml}) / \mathrm{NBT}(4.5 \mu \mathrm{l} / \mathrm{ml})$ solution (cat. no. 0885/0329; Amresco, Inc.) in a humidified dark chamber at room temperature, overnight. Subsequently, the sections were counterstained with $10 \%$ nuclear fast red (Gaide Chemical reagent Company, Shanghai, China). Following dehydration in ascending concentrations of ethanol and xylene, the sections were mounted in mounting medium (Vector Laboratories, Inc., Burlingame, CA, USA). Positive controls and no-probe controls were included for each hybridization procedure. The slides were then observed and the images captured using an Olympus X71 optic microscope (Olympus Corporation, Tokyo, Japan).

Statistical analysis. Statistical analyses were performed using the statistical package SPSS version 19.0 (IBM SPSS, Armonk, NY, USA). Associations between the expression of miRNA and clinicopathological variables were assessed using Mann-Whitney or Kruskal-Wallis analyses. Groups were compared using a Pearson $\chi^{2}$ test. Kaplan-Meier analysis was used to analyze survival rates, and a log-rank test was used to compare survival curves. Multivariate analysis was performed using Cox proportional hazards regression analysis. All statistical tests were two-sided. $\mathrm{P}<0.05$ was considered to indicate a statistically significant difference.

\section{Results}

miRNA expression patterns. Of the 768 miRNAs analyzed in the microarray, 102 miRNAs were differentially expressed in HGSC, compared with CCC, and 83 unique miRNAs retained significance $(\mathrm{P}<0.015)$, with at least a 2 -fold difference, following correction for multiple comparisons. In total, 33 miRNAs were upregulated and 50 were downregulated in the HGSC samples, compared with the CCC samples (Fig. 1; Table II). The results of the unsupervised hierarchical clustering, based on the expression of the significantly differentially expressed miRNAs are shown in Fig. 1.

Validation of unique miRNAs. To confirm the miRNA expression pattern obtained from microarray analysis, RT-qPCR was used to quantify the expression levels of specific miRNAs. In total, four of the 83 miRNAs (miR-510, miR-129-3p, miR-483 and miR-449a) were differentially expressed in HGSC and $\mathrm{CCC}$, and were selected for further validation using RT-qPCR. The clinicopathological characteristics of the patients with OC in the validation cohort are shown in Table I. These miRNAs were selected as $>5$-fold changes in expression levels were observed in patients with OSC at stage I, compared with stage III $(\mathrm{P}<0.005)$. miR-510, miR-129-3p, miR-483 and miR-449a were among the most significantly differentially expressed miRNAs between HGSC and CCC. The RT-qPCR results revealed that miR-510 and miR-129-3p were significantly downregulated, and miR-483 and miR-449a were significantly upregulated in CCC, compared with HGSC $(\mathrm{P}<0.05)$, which was consistent with the results of the microarray. Fig. 2 lists the miRNA expression levels in patients with OSC at different stages, according to the microarray and RT-qPCR data.
Correlation between the expression of miRNA, patient clinicopathological data and survival rates. The clinicopathological features and prognosis of all the patients with ovarian cancer were obtained from the hospital records. The follow-up duration was between 1 and 104 months (mean, 49 months). During the follow-up period, 29 of the 78 patients $(37.1 \%)$ succumbed to mortality. The clinicopathological features included age $(>50$ or $\leq 50$ years), subtype (CCC or HGSC), FIGO stage, lymphatic metastasis (negative or positive) and chemotherapy sensitivity (complete or incomplete response). Data regarding sensitivity to chemotherapy were only available for patients with HGSC.

The RT-qPCR results for miR-510, miR-129-3p, miR-483 and $\mathrm{miR}-449 \mathrm{a}$ were first separated into high and low expression, defined according to the median value of the miRNA levels in the tumor samples. Mann-Whitney and Kruskal-Wallis tests were performed to analyze the expression levels of miR-510, miR-129-3p, miR-483 and miR-449a in different age groups ( $>50$ or $\leq 50$ years), stages of lymph node metastasis and FIGO stage. All four miRNAs were significantly associated with the FIGO stage $(\mathrm{P}<0.01)$ (Table III). The upregulation of miR-483 was also associated with positive tumor lymphatic metastasis $(\mathrm{P}<0.01)$. The expression of these miRNAs was not associated with the age of the patient $(\mathrm{P}>0.05)$ (Table III).

Clinicopathological features and the expression of miRNAs, including miR-510, miR-129-3p, miR-483 and miR-449a, were included in the univariate survival analysis. Univariate analysis revealed that FIGO stage, subtype of ovarian cancer, chemosensitivity, lymphatic metastasis status, and expression levels of miR-510 and miR-129-3p were associated with prognosis $(\mathrm{P}<0.05)$, whereas the age of the patient and the expression levels of miR-449a and miR-483-5p were not ( $\mathrm{P}>0.05$; Table IV). Downregulation in the expression levels of miR-510 and miR-129-3p were clearly associated with poor prognosis (Fig. 3).

Expression levels of miR-510 in the normal control, HGSC, LGSC and CCC tissue samples. miR-510 was identified among the most significantly altered miRNAs between the HGSC and CCC tissue samples. Our previous study (20) also revealed its prognostic value for OSC. In order to evaluate the expression of miR-510 in ovarian tumors, the expression of miR-510 was detected in a cohort of patients with ovarian cancer, including 42 cases of HGSC, 10 cases of LGSC and 36 cases of CCC. In addition, 10 samples of normal ovarian tissue, which comprised ovarian surface epithelium (OSE), were selected as the control. The results revealed that the expression levels of miR-510 were significantly higher in the CCC and LGSC specimens, compared with the OSE and HGSC specimens. Although the mean value of miR-510 expression in the HGSC samples was marginally lower than that in the OSE samples, no significant difference was identified between these two groups (Fig. 4). No significant difference was observed in the expression of miR-510 between the CCC and LGSC samples.

ISH detection of miR-510 in LGSC, HGSC and CCC. To determine the location of miR-510 in EOC, the expression levels of miR-510 were qualitatively detected in the HGSC, LGSC and CCC specimens using ISH (Fig. 5). The results revealed that miR-510 was densely distributed in the malignant cells, particularly in the cytoplasm and nuclei of the LGSC (Fig 5A) 
Table II. List of miRNAs identified to be differentially expressed in HGSC and CCC samples.

\begin{tabular}{|c|c|c|c|}
\hline $\operatorname{miR}$ & P-value & $\mathrm{FC}$ & $\mathrm{H}$ \\
\hline hsa-miR-510 & $3.56 \mathrm{E}-05$ & 34.14 & $\mathrm{CCC}$ \\
\hline hsa-miR-129-3p & $9.27 \mathrm{E}-04$ & 30.50 & $\mathrm{CCC}$ \\
\hline hsa-miR-330-5p & 1.91E-04 & 17.08 & $\mathrm{CCC}$ \\
\hline hsa-miR-500* & $1.11 \mathrm{E}-03$ & 15.96 & $\mathrm{CCC}$ \\
\hline hsa-miR-493* & $1.75 \mathrm{E}-04$ & 9.93 & $\mathrm{CCC}$ \\
\hline hsa-miR-129-5p & $1.04 \mathrm{E}-03$ & 9.80 & $\mathrm{CCC}$ \\
\hline hsa-miR-299-5p & $1.21 \mathrm{E}-03$ & 8.67 & $\mathrm{CCC}$ \\
\hline hsa-miR-29b-2* & $2.81 \mathrm{E}-07$ & 7.94 & $\mathrm{CCC}$ \\
\hline hsa-miR-885-5p & 4.31E-06 & 6.92 & $\mathrm{CCC}$ \\
\hline hsa-miR-486-5p & $1.54 \mathrm{E}-03$ & 6.64 & $\mathrm{CCC}$ \\
\hline hsa-miR-98 & $1.23 \mathrm{E}-02$ & 6.44 & $\mathrm{CCC}$ \\
\hline hsa-miR-220a & $1.86 \mathrm{E}-03$ & 6.39 & $\mathrm{CCC}$ \\
\hline hsa-miR-505* & 3.61E-09 & 6.17 & $\mathrm{CCC}$ \\
\hline hsa-miR-30a* & $3.67 \mathrm{E}-10$ & 6.07 & $\mathrm{CCC}$ \\
\hline hsa-miR-505 & $5.62 \mathrm{E}-06$ & 6.01 & $\mathrm{CCC}$ \\
\hline hsa-miR-154* & $5.74 \mathrm{E}-03$ & 5.99 & $\mathrm{CCC}$ \\
\hline hsa-miR-379* & $6.01 \mathrm{E}-03$ & 5.79 & $\mathrm{CCC}$ \\
\hline hsa-miR-629 & $3.60 \mathrm{E}-03$ & 5.64 & $\mathrm{CCC}$ \\
\hline hsa-miR-9 & 2.61E-04 & 5.42 & $\mathrm{CCC}$ \\
\hline hsa-miR-496 & $2.75 \mathrm{E}-03$ & 5.39 & $\mathrm{CCC}$ \\
\hline hsa-miR-30e & $1.24 \mathrm{E}-10$ & 5.19 & $\mathrm{CCC}$ \\
\hline hsa-miR-616 & $6.15 \mathrm{E}-04$ & 5.19 & $\mathrm{CCC}$ \\
\hline hsa-miR-376a* & $1.22 \mathrm{E}-02$ & 4.98 & $\mathrm{CCC}$ \\
\hline hsa-miR-9* & $4.55 \mathrm{E}-05$ & 4.86 & $\mathrm{CCC}$ \\
\hline hsa-miR-30c- $1^{*}$ & $6.26 \mathrm{E}-03$ & 4.79 & $\mathrm{CCC}$ \\
\hline hsa-miR-33a* & $1.43 \mathrm{E}-03$ & 4.61 & $\mathrm{CCC}$ \\
\hline hsa-miR-382 & $1.77 \mathrm{E}-03$ & 4.59 & $\mathrm{CCC}$ \\
\hline hsa-miR-206 & $1.95 \mathrm{E}-04$ & 4.59 & $\mathrm{CCC}$ \\
\hline hsa-miR-107 & $2.17 \mathrm{E}-03$ & 4.33 & $\mathrm{CCC}$ \\
\hline hsa-miR-424* & 4.93E-03 & 4.31 & $\mathrm{CCC}$ \\
\hline hsa-miR-31 & $7.59 \mathrm{E}-03$ & 3.13 & $\mathrm{CCC}$ \\
\hline hsa-let-7g ${ }^{*}$ & $1.17 \mathrm{E}-04$ & 3.12 & $\mathrm{CCC}$ \\
\hline hsa-miR-30a & $2.21 \mathrm{E}-09$ & 3.00 & $\mathrm{CCC}$ \\
\hline hsa-miR-30c & $3.27 \mathrm{E}-05$ & 2.95 & $\mathrm{CCC}$ \\
\hline hsa-miR-345 & $3.71 \mathrm{E}-05$ & 2.91 & $\mathrm{CCC}$ \\
\hline hsa-miR-190 & $2.02 \mathrm{E}-05$ & 2.91 & $\mathrm{CCC}$ \\
\hline hsa-miR-628-3p & $1.21 \mathrm{E}-03$ & 2.84 & $\mathrm{CCC}$ \\
\hline hsa-miR-30b & $1.87 \mathrm{E}-04$ & 2.54 & $\mathrm{CCC}$ \\
\hline hsa-miR-502-3p & $6.07 \mathrm{E}-04$ & 2.53 & $\mathrm{CCC}$ \\
\hline hsa-miR-497 & $3.19 \mathrm{E}-03$ & 2.46 & $\mathrm{CCC}$ \\
\hline hsa-miR-744 & $1.46 \mathrm{E}-04$ & 2.42 & $\mathrm{CCC}$ \\
\hline hsa-miR-455-5p & $1.15 \mathrm{E}-03$ & 2.30 & $\mathrm{CCC}$ \\
\hline hsa-miR-532-5p & $9.70 \mathrm{E}-04$ & 2.27 & $\mathrm{CCC}$ \\
\hline hsa-miR-592 & $2.81 \mathrm{E}-03$ & 2.24 & $\mathrm{CCC}$ \\
\hline hhsa-miR-203 & $2.16 \mathrm{E}-03$ & 2.25 & $\mathrm{CCC}$ \\
\hline hsa-miR-30d & $5.73 \mathrm{E}-06$ & 2.16 & $\mathrm{CCC}$ \\
\hline hsa-miR-502-5p & $5.56 \mathrm{E}-03$ & 2.15 & $\mathrm{CCC}$ \\
\hline hsa-miR-532-3p & 4.64E-03 & 2.14 & $\mathrm{CCC}$ \\
\hline hsa-miR-423-5p & $3.11 \mathrm{E}-03$ & 2.10 & $\mathrm{CCC}$ \\
\hline hsa-miR-34a* & 3.79E-04 & 2.06 & $\mathrm{CCC}$ \\
\hline
\end{tabular}

Table II. Continued.

\begin{tabular}{lccc}
\hline miR & P-value & FC & H \\
\hline hsa-miR-19b-1 $^{*}$ & $5.07 \mathrm{E}-04$ & 0.49 & HGSC \\
hsa-miR-20a* & $1.45 \mathrm{E}-04$ & 0.49 & HGSC \\
hsa-miR-141 & $1.06 \mathrm{E}-02$ & 0.47 & HGSC \\
hsa-miR-7-1* & $6.31 \mathrm{E}-03$ & 0.48 & HGSC \\
hsa-miR-380* & $1.51 \mathrm{E}-04$ & 0.46 & HGSC \\
hsa-miR-222 & $7.36 \mathrm{E}-03$ & 0.46 & HGSC \\
hsa-miR-218 & $1.18 \mathrm{E}-03$ & 0.44 & HGSC \\
hsa-miR-149 & $6.58 \mathrm{E}-03$ & 0.44 & HGSC \\
hsa-miR-135b* & $4.38 \mathrm{E}-04$ & 0.41 & HGSC \\
hsa-miR-378 & $5.30 \mathrm{E}-05$ & 0.40 & HGSC \\
hsa-miR-604 & $1.12 \mathrm{E}-02$ & 0.40 & HGSC \\
hsa-miR-422a & $3.66 \mathrm{E}-03$ & 0.40 & HGSC \\
hsa-miR-146b-3p & $1.91 \mathrm{E}-03$ & 0.39 & HGSC \\
hsa-miR-187 & $1.14 \mathrm{E}-02$ & 0.39 & HGSC \\
hsa-miR-190b & $9.19 \mathrm{E}-04$ & 0.36 & HGSC \\
hsa-miR-99a* & $5.84 \mathrm{E}-03$ & 0.36 & HGSC \\
hsa-miR-922 & $1.10 \mathrm{E}-04$ & 0.32 & HGSC \\
hsa-miR-146b-5p & $8.80 \mathrm{E}-05$ & 0.29 & HGSC \\
hsa-miR-582-5p & $5.03 \mathrm{E}-03$ & 0.22 & HGSC \\
hsa-miR-34c-5p & $2.12 \mathrm{E}-04$ & 0.19 & HGSC \\
hsa-miR-639 & $1.68 \mathrm{E}-03$ & 0.17 & HGSC \\
hsa-miR-130a* & $1.14 \mathrm{E}-02$ & 0.16 & HGSC \\
hsa-miR-19a* & $1.39 \mathrm{E}-03$ & 0.13 & HGSC \\
hsa-miR-135a & $9.89 \mathrm{E}-07$ & 0.12 & HGSC \\
hsa-miR-944 & $2.69 \mathrm{E}-04$ & 0.12 & HGSC \\
hsa-miR-124 & $2.74 \mathrm{E}-05$ & 0.11 & HGSC \\
hsa-miR-551a & $3.72 \mathrm{E}-03$ & 0.10 & HGSC \\
hsa-miR-182* & $6.17 \mathrm{E}-03$ & 0.083 & HGSC \\
hsa-miR-412 & $1.70 \mathrm{E}-06$ & 0.069 & HGSC \\
hsa-miR-483-5p & $8.81 \mathrm{E}-07$ & 0.068 & HGSC \\
hsa-miR-34b* & $1.01 \mathrm{E}-06$ & 0.038 & HGSC \\
hsa-miR-449b & $9.52 \mathrm{E}-05$ & 0.031 & HGSC \\
hsa-miR-449a & $2.27 \mathrm{E}-07$ & 0.028 & HGSC \\
\hline & & & \\
\hline
\end{tabular}

$\mathrm{H}$, sample associated with high expression levels; FC, fold change; CCC, clear cell carcinoma; HGSC, high-grade serous carcinoma; miR, microRNA.

and CCC (Fig. 5B) samples. The positive signal of miR-510 was poorly expressed in the malignant cells of the HGSC samples (Fig. 5C), compared with the LGSC and CCC samples.

\section{Discussion}

In the present study, a number of miRNAs were identified distinguishing HGSC from CCC, and differential miRNA expression was associated with histological type and stage, as well as overall survival rates. The expression levels of miR-510 were further examined in samples of normal ovarian tissue and ovarian tumor tissue, including HGSC, LGSC and CCC, using RT-qPCR and ISH. The expression levels of miR-510 wereupregulated in the low-grade tumor samples (LGSC and CCC) 


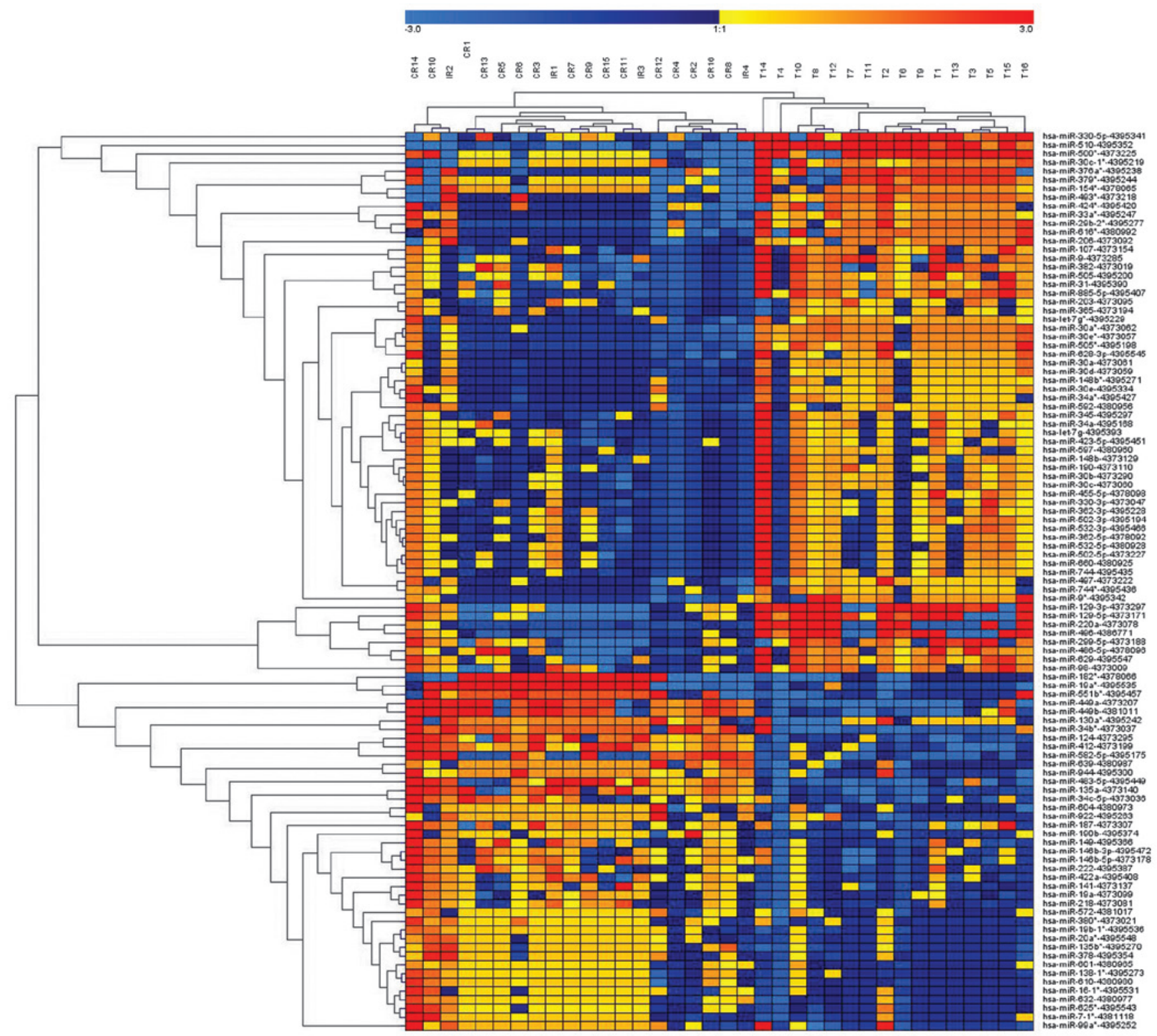

Figure 1. Unsupervised hierarchical clustering of miRNAs and samples from the microarray data. The miRNA clustering tree is on the left and the sample clustering tree is above. The color scale at the top indicates the relative expression level of an miRNA across all samples. Red indicates that the expression level is higher than the mean; blue indicates that the expression level is lower than the mean. Each row represents one miRNA and each column represents one sample. Clustering was performed using $\log 10$ (fold change) ratios, which passed the filtering criteria across all samples (standard deviation $<1.0$ ). The heat map contained 102 unique miRNAs, which were differentially expressed in patients with high-grade serous carcinoma and clear cell carcinoma.

and downregulated in the high-grade tumor samples (HGSC), compared with the normal ovarian tissue samples. To the best of our knowledge, there are few previous reports regarding the differentially expressed miRNAs between HGSC and CCC, and the present study is the first study to investigate the expression of miR-510 in HGSC, LGSC and CCC, compared with EOC.

In the present study, 33 upregulated and 50 downregulated miRNAs were identified in HGSC compared with CCC, using a microarray. Of the 83 key miRNAs identified in the present study, four (miR-510, miR-129-3p, miR-483 and miR-449a) were validated using RT-qPCR, and their expression levels were confirmed to be consistent with the microarray data. The results indicated that the differential miRNA pattern in HGSC, compared with CCC, was credible. These data on the expression of miRNAs in HGSC and CCC are consistent with what has been reported in previous literature $(21,22)$. The pattern of miRNA expression distinguishing HGSC from CCC has not been widely investigated. Vilming Elgaaen et al found that 28 miRNAs are upregulated and 50 miRNAs are downregulated in HGSC $(n=12)$, compared with CCC $(n=9)$. Their dysregulated miRNA expression profiles shared certain key miRNAs with the results of the present study (Table V) (21). Heejeong measured eight miRNAs in ovarian cancer samples using RT-qPCR, and reported that the expression levels of miR-30a-3p, miR-30c and miR-30e-3p were significantly higher in CCC samples than in HGSC samples (22). Higher expression levels of miR-181d, miR-30c, miR-30d and miR-30e-3p were associated withsignificantly improved disease-free and overall survival rates, and miR-30a-3p, miR-30c and miR-30e-3p may regulate the ovarian carcinoma-specific gene, CDH13 (22). 

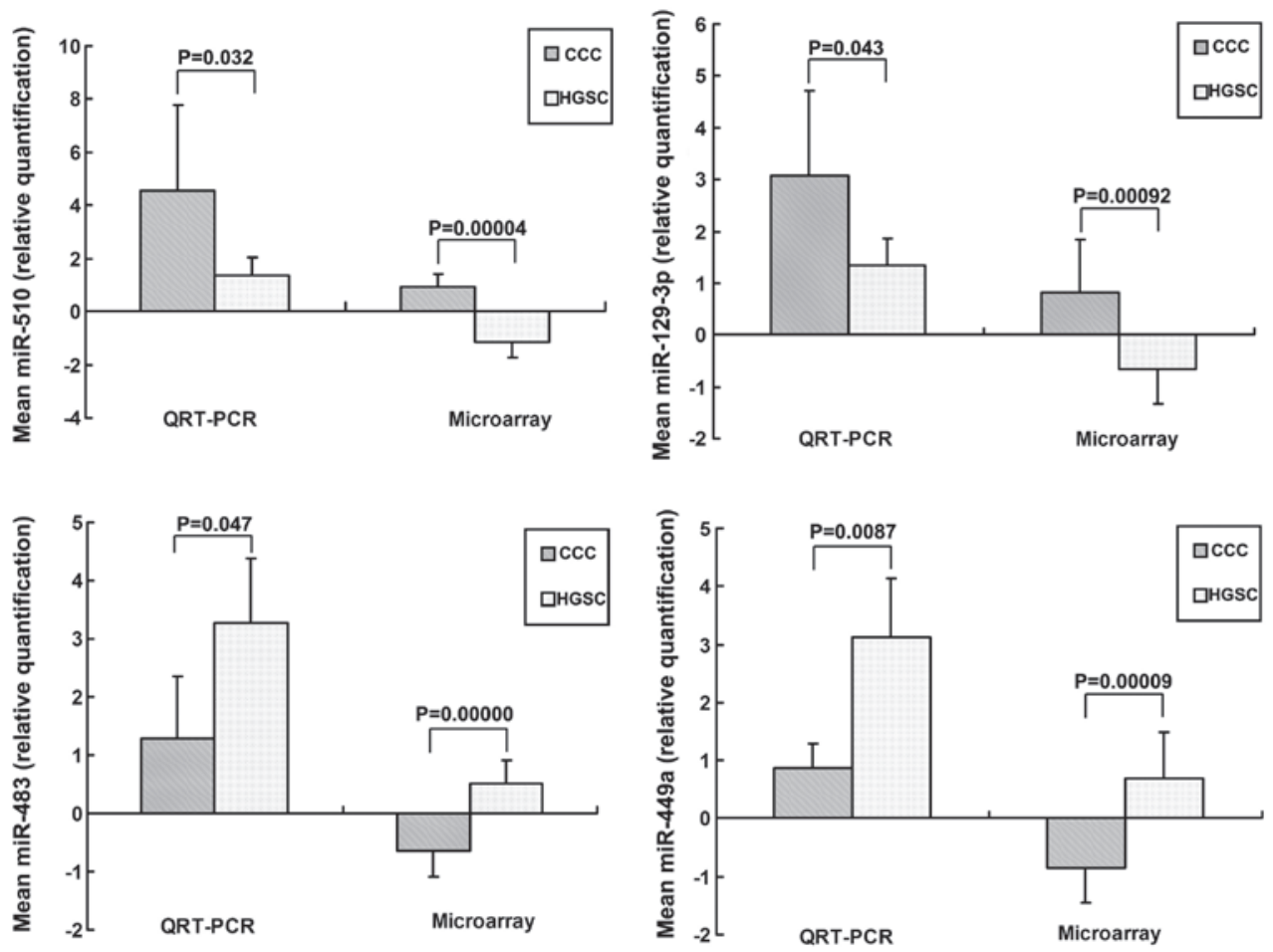

Figure 2. Comparison between RT-qPCR results and microarray results. The transverse bars indicate the median value, error bars indicate the SD, microarray results are presented as $\log 10^{2}$-averageDC and the data are expressed as the mean \pm SD of HGSC $(n=20)$ and CCC $(n=6)$. The RT-qPCR data are presented as the logarithmic mean expression level relative to HGSC, and the data are expressed as the mean \pm SD of HGSC ( $n=42)$ and CCC ( $n=36)$. Each sample was assessed in triplicate. Statistical significance was determined using a Mann-Whitney test. The microarray and RT-qPCR results revealed significant differences between HGSC and CCC. The expression levels of miR-510 and miR-129-3p were significantly downregulated, those of and miR-483-5p and miR-449a were significantly upregulated in HGSC, compared with CCC $(\mathrm{P}<0.05)$. RT-qPCR, reverse transcription-quantitative polymerase chain reaction; CCC, clear cell carcinoma; HGSC, high-grade serous carcinoma; SD, standard deviation.
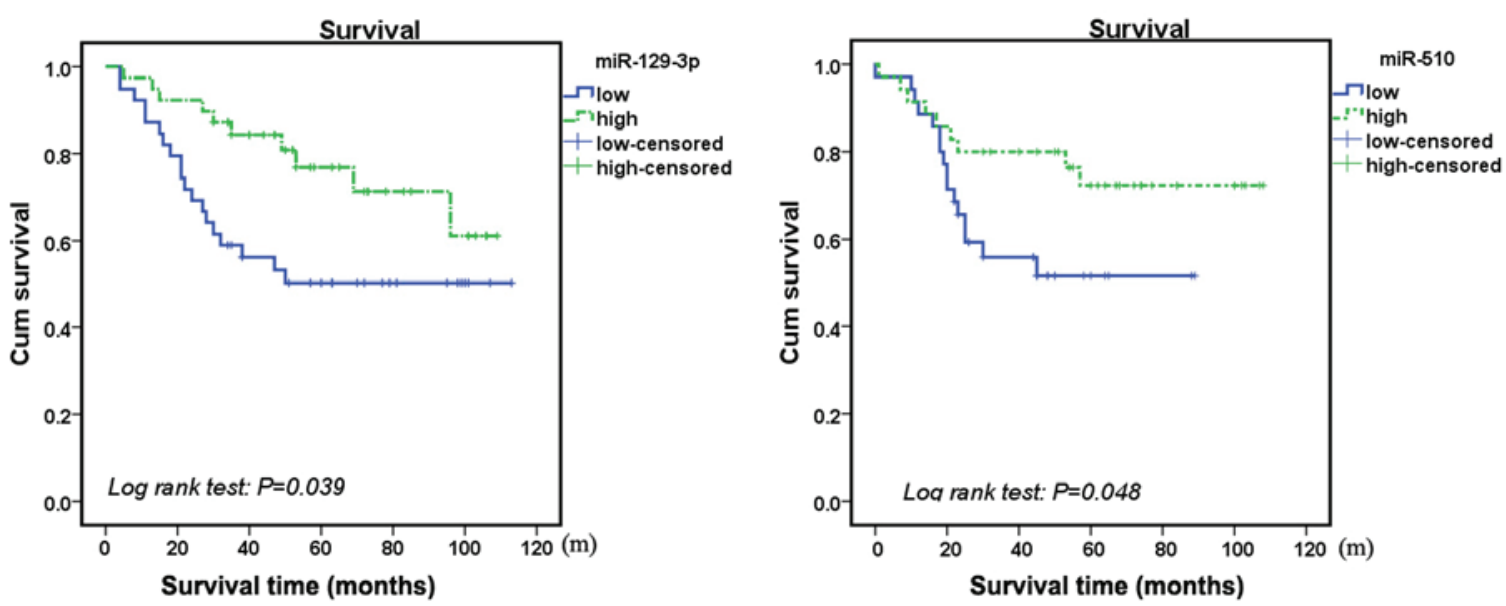

Figure 3. Kaplan-Meier curves of the overall survival rates of patients with EOC, stratified by expression levels of miR-510 and miR-129-3p. Reverse transcription-quantitative polymerase chain reaction data of miR-510 and miR-129-3p were separated into high and low expression values. miR-129-3p was separated by the median value of the miRNA levels in the tumor samples. MiR-510 was separated by the expression level which can give the greatest significance in log-rank test in the Kaplan-Meier analysis. The reference was 2 for miR-510. The patients with EOC were divided into groups exhibiting high expression (solid line) and low expression (dotted line) levels of miR-510 and miR-129-3p. The survival duration of the patients with EOC was associated with miRNA expression levels. Lower expression levels of miR-510 ( $\mathrm{P}=0.048)$ and miR-129-3p $(\mathrm{P}=0.039)$ were associated with poorer overall disease-specific survival rates. EOC, epithelial ovarian cancer; miR, microRNA; Cum, cumulative. High, high expression level group; low, low expression level group.

Of the dysregulated miRNAs identified in the present study, certain miRNAs were associated with a specific subtype of EOC. The upregulation of miR-29b, miR-30a, miR-486-5p and miR-30e, and the downregulation of miR-20a were specific to the CCC samples. The upregulation of miR-7, miR-22,
miR-302b, miR-373, miR-34c-5p, miR-449a and miR-146b-5p, and the downregulation of miR-148b, miR-31 and miR-211 are reported to be specific to serous carcinoma (21). The miRNAs differentially expressed in the HGSC and CCC samples may be associated with the different carcinogenesis pathways, as 
Table III. Correlation between the expression levels of miR-510, miR-129-3p, miR-449a and miR-483, and clinicopathological features of ovarian carcinomas.

\begin{tabular}{|c|c|c|c|c|c|c|c|c|c|c|c|c|c|}
\hline \multirow{2}{*}{$\begin{array}{l}\text { Clinicopathological } \\
\text { features }\end{array}$} & \multirow[b]{2}{*}{$\mathrm{n}$} & \multicolumn{2}{|c|}{ miR-483 } & \multirow[b]{2}{*}{$\mathrm{P}$} & \multicolumn{2}{|c|}{ miR-510 } & \multirow[b]{2}{*}{$\mathrm{P}$} & \multicolumn{2}{|c|}{ miR-129-3p } & \multirow[b]{2}{*}{$\mathrm{P}$} & \multicolumn{2}{|c|}{$\operatorname{miR}-449 a$} & \multirow[b]{2}{*}{$\mathrm{P}$} \\
\hline & & $\mathrm{L}$ & $\mathrm{H}$ & & $\mathrm{L}$ & $\mathrm{H}$ & & $\mathrm{L}$ & $\mathrm{H}$ & & $\mathrm{L}$ & $\mathrm{H}$ & \\
\hline Age in years & & & & 0.247 & & & 0.224 & & & 0.882 & & & 0.458 \\
\hline$<50$ & 41 & 24 & 17 & & 20 & 21 & & 22 & 19 & & 22 & 19 & \\
\hline$\geq 50$ & 37 & 15 & 22 & & 19 & 18 & & 17 & 18 & & 17 & 20 & \\
\hline Subtypes & & & & 0.000 & & & & & & 0.002 & & & 0.000 \\
\hline HGSC & 42 & 8 & 34 & & 33 & 9 & & 31 & 11 & & 8 & 34 & \\
\hline $\mathrm{CCC}$ & 36 & 31 & 5 & & 6 & 30 & & 8 & 28 & & 31 & 5 & \\
\hline FIGO stage & & & & 0.001 & & & 0.001 & & & 0.010 & & & 0.001 \\
\hline I & 33 & 28 & 5 & & 6 & 27 & & 8 & 25 & & 28 & 5 & \\
\hline II & 4 & 2 & 2 & & 2 & 2 & & 0 & 4 & & 2 & 2 & \\
\hline III & 36 & 9 & 30 & & 29 & 10 & & 29 & 10 & & 9 & 30 & \\
\hline IV & 2 & 0 & 2 & & 2 & 0 & & 2 & 0 & & 0 & 2 & \\
\hline LN metastasis & & & & 0.048 & & & 0.274 & & & 0.173 & & & 0.084 \\
\hline Absent & 50 & 29 & 21 & & 23 & 27 & & 22 & 28 & & 30 & 20 & \\
\hline Present & 28 & 10 & 18 & & 16 & 12 & & 17 & 11 & & 9 & 19 & \\
\hline
\end{tabular}

miR, microRNA; n, number; P, P-value; L, low expression levels; H, high expression levels; FIGO stage, International Federation of Gynecology and Obstetrics stage; HGSC, high-grade serous carcinoma; CCC, clear cell carcinoma; LN, lymph node. P-values indicated in bold are considered statistically significant at $\mathrm{P}<0.05$.

well as their distinct morphological or genetic features. Thhese miRNAs were differentially expressed in different histological types of ovarian carcinoma, which is pertinent to the fact that different histological types are biologically and pathogenetically distinct entities.

The association between the expression levels of miR-510, miR-129-3p, miR-483 and miR-449a and clinicopathological features and prognosis, were also investigated, and it was revealed that all were associated with the FIGO stage. In addition, miR-483 was associated with the lymphatic metastasis status, and lower expression levels of miR-510 and miR-129-3p were associated with a poor prognosis. The majority of the HGSC samples were at an advanced stage, whereas the CCC samples were at stage I or II. The miRNAs differentially expressed in the HGSC and CCC samples may also be associated with the progression and FIGO stage of EOC.

miR-510 is one of the miRNAs, which most clearly distinguished between the CCC and HGSC samples in the prsent study. Our previous study demonstrated that higher expression levels of miR-510 in stage I OSC, compared with stage III OSC were associated with survival rates. This suggested that miR-510 may be important in EOC. In order to further investigate the role of miR-510 in EOC, the expression of miR-510 was quantitatively and qualitatively examined using RT-qPCR and ISH in samples, including normal ovarian tissue, LGSC, HGSC and CCC. The results revealed that the expression of levels of miR-510 in CCC and LGSC were significantly higher than those in HGSC and OSE. Although the mean expression value of miR-510 in HGSC was lower than that in OSE, no difference was identified between these two groups. Additionally, no difference was identified between the expression levels of miR-510 in the CCC and LGSC samples. ISH confirmed that miR-510 was expressed in the cancer cells. These results suggested an important and complicated role of miR-510 in EOC.

To the best of our knowledge, few studies have focused on miR-510, and their results were ambiguous (18,22-24). miR-510 belongs to the miR-506-514 gene cluster, which includes seven distinct miRNAs: miR-506,-507,-508,-509,-510,-513 and -514 , and has been previously reported to be conserved in primates $(24,25)$. This gene cluster is located at Xq27.3, a chromosomal region associated with Fragile X syndrome, and female patients with Fragile X syndrome suffer from primary ovarian insufficiency (24). In serous ovarian carcinoma, it has been reported that patients with low expression levels of the chrXq27.3 miRNA cluster experience shorter progression-free survival rates, and downregulation of chrXq27.3 miRNA was a possible independent prognostic indicator of early relapse (26). By analyzing the miRNA profile in TGCA data, the miRNAs located at Xq27.3 have been revealed to be members of a highly correlated and co-expressed miRNA cluster (24). Our previous study demonstrated that five members of this gene cluster, including miR-510, miR-513a-3p, miR-509-3p, miR-508-3p and miR-509-5p, were the most differentially expressed miRNA between stage I and stage III serous ovarian carcinoma. Low expression levels of miR-510 and miR-509 was associated with poor prognosis (20). Bente et al (21) found that miR-509-3-5p, miR-509-5p, miR-509-3p and miR-510 were the most significant differentiators between HGSC and $\mathrm{CCC}$, all of which were significantly overexpressed in CCC, compared with HGSC. The expression levels of these miRNAs were higher in CCC and lower in HGSC, compared with OSE, which was consistent with the present study, with the exception 
Table IV. Univariate analysis of expression and overall cancer survival in subjects with ovarian serous carcinoma.

\begin{tabular}{|c|c|c|c|c|c|c|}
\hline \multirow{2}{*}{$\begin{array}{l}\text { Clinicopathological } \\
\text { parameter }\end{array}$} & \multirow[b]{2}{*}{$\mathrm{n}$} & \multirow{2}{*}{$\begin{array}{l}\text { Succumbed to } \\
\text { mortality (n) }\end{array}$} & \multirow{2}{*}{$\begin{array}{l}\text { Survival rate } \\
\quad \text { (mean) }\end{array}$} & \multicolumn{2}{|c|}{$\begin{array}{l}\text { Survival rate } \\
(95 \% \mathrm{CI})\end{array}$} & \multirow[b]{2}{*}{ P-value } \\
\hline & & & & Lower & Upper & \\
\hline Age & & & & & & 0.461 \\
\hline$<50$ & 41 & 14 & 82.977 & 70.037 & 95.917 & \\
\hline$\geq 50$ & 37 & 15 & 71.944 & 58.239 & 85.649 & \\
\hline Subtype & & & & & & 0.000 \\
\hline HGSC & 42 & 24 & 61.417 & 47.714 & 75.119 & \\
\hline $\mathrm{CCC}$ & 36 & 5 & 97.581 & 88.214 & 106.948 & \\
\hline LN metastasis & & & & & & 0.000 \\
\hline- & 50 & 11 & 94.426 & 84.191 & 104.661 & \\
\hline+ & 28 & 18 & 49.412 & 35.012 & 63.812 & \\
\hline $\begin{array}{l}\text { Chemosensitivity } \\
\text { (only available in HGSC) }\end{array}$ & & & & & & 0.022 \\
\hline $\mathrm{CR}$ & 26 & 12 & 71.410 & 54.913 & 87.907 & \\
\hline IR & 16 & 12 & 37.313 & 20.048 & 54.577 & \\
\hline FIGO stage & & & & & & 0.000 \\
\hline I & 33 & 2 & 103.131 & 95.338 & 110.925 & \\
\hline II & 4 & 2 & 67.500 & 44.585 & 90.415 & \\
\hline III & 39 & 23 & 59.919 & 45.488 & 74.349 & \\
\hline IV & 2 & 2 & 12.500 & 12.000 & 29.160 & \\
\hline miR-510 & & & & & & 0.048 \\
\hline Low-expression & 41 & 19 & 62.972 & 43.206 & 82.738 & \\
\hline High expression & 37 & 10 & 91.875 & 75.252 & 108.498 & \\
\hline \multicolumn{7}{|l|}{ miR-129-3p } \\
\hline Low expression & 39 & 20 & 61.294 & 40.391 & 82.198 & 0.039 \\
\hline High expression & 39 & 9 & 90.056 & 72.990 & 107.121 & \\
\hline \multicolumn{7}{|l|}{$\operatorname{miR}-483$} \\
\hline Low expression & 39 & 10 & 90.722 & 73.877 & 107.567 & 0.083 \\
\hline High expression & 39 & 19 & 60.471 & 39.541 & 81.400 & \\
\hline \multicolumn{7}{|l|}{$\mathrm{miR}-449 \mathrm{a}$} \\
\hline Low expression & 39 & 11 & 88.647 & 70.241 & 107.053 & 0.198 \\
\hline High expression & 39 & 18 & 61.444 & 42.643 & 80.246 & \\
\hline
\end{tabular}

CI, confidence interval; HGSC, high-grade serous ovarian carcinoma; CCC, clear cell ovarian carcinoma; CR, complete response; IR, incomplete response; LN, lymph node.

that the present study did not identify a significant difference in the expression of miR-510 between HGSC and OSE (21). These results suggested that miR-510 has different roles in different subtypes of ovarian cancer. The upregulation of miR-510 suggested that miR-510 may act as an oncogene in LGSC and CCC. The fact that miR-510 was downregulated in HGSC, and low expression levels were associated with early relapse, suggested that miR-510 may act as a tumor suppressor or be due to the high gene instability of HGSC.

Increasing numbers of studies have demonstrated that the different subtypes of ovarian carcinoma represent distinct disease entities, rather than different manifestations of one disease (7,27). Novel histopathological, molecular and genetic studies have developed an improved model for ovarian carci- nogenesis, revealing at least two broad categories, type I and type II. LGSC and CCC are type I tumors, which are considered to behave in an indolent manner, and appear to evolve in a stepwise fashion between ovarian epithelial inclusions, benign cystadenomas and borderline tumors. They are often confined to the ovary at the point of diagnosis, with a stable genome and without TP53 mutations. HGSC is a type II tumor, which is considered to be more aggressive. It is often diagnosed at an advanced stage and is genetically unstable; the majority exhibiting TP53 mutations, and almost half of the cases exhibit abnormalities in BRCA1/2 $(7,28)$.

HGSC and LGSC are currently known to be the products of two completely disparate tumorigenic pathways, with only rare intersection and distinct differences in prognosis and 
Table V. Overlapping findings in published data and the present study of compared differential miRNA profiling between HGSC and CCC (21).

\begin{tabular}{|c|c|}
\hline Expression level & miRNA \\
\hline High in HGSC & $\begin{array}{l}\text { hsa-miR-135b* } \\
\text { hsa-miR-141 } \\
\text { hsa-miR-20a* } \\
\text { hsa-miR-378 } \\
\text { hsa-miR-99a* }\end{array}$ \\
\hline High in CCC & $\begin{array}{l}\text { hsa-miR-154* } \\
\text { hsa-miR-29b-2* } \\
\text { hsa-miR-299-5p } \\
\text { hsa-miR-362-5p } \\
\text { hsa-miR-376a* } \\
\text { hsa-miR-379* } \\
\text { hsa-miR-424* } \\
\text { hsa-miR-493* } \\
\text { hsa-miR-500* } \\
\text { hsa-miR-502-3p } \\
\text { has-miR-510 } \\
\text { hsa-miR-532-3p } \\
\text { hsa-miR-532-5p } \\
\text { hsa-miR-885-5p }\end{array}$ \\
\hline
\end{tabular}

HGSC, high-grade serous carcinoma; CCC, clear cell carcinoma; miR, microRNA.

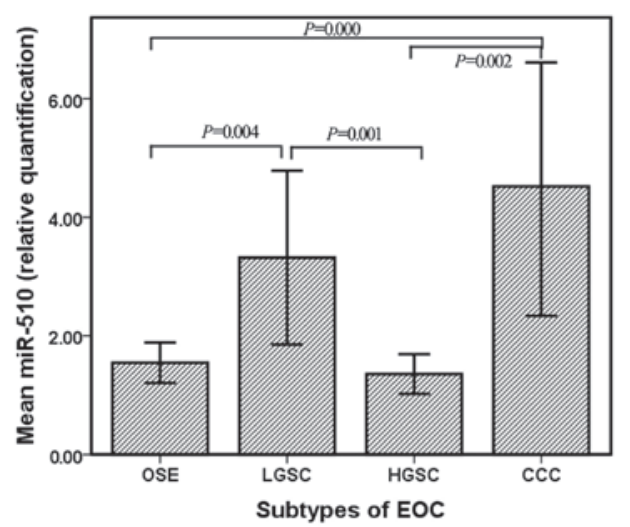

Figure 4. Expression levels of miR-510 in normal ovarian tissue, HGSC, LGSC and CCC samples. The transverse bars indicate the median value of the results of the reverse transcription-quantitative polymerase chain reaction of miR-510, error bars indicate $95 \%$ confidential intervala. The expression levels of miR-510 in the CCC and LGSC samples were significantly higher, compared with the normal ovarian tissue and HGSC samples. No significan difference was identified between the expression levels of miR-510 in the CCC and LGSC samples $(\mathrm{P}=0.198)$ or the HGSC and normal ovarian tissue samples $(\mathrm{P}=0.860)$. miR, microRNA; EOC, epithelial ovarian cancer; HGSC, high-grade serous carcinoma; CCC, clear cell carcinoma; LGSC, low-grade serous carcinoma; OSE, ovarian surface epithelium.

chemotherapeutic sensitivity (28). This corresponds with the results of the present study, demonstrating the upregulation of miR-510 in CCC and LGSC, and the novel histopathological model that CCC and LGSC belong to type I. Downregulation
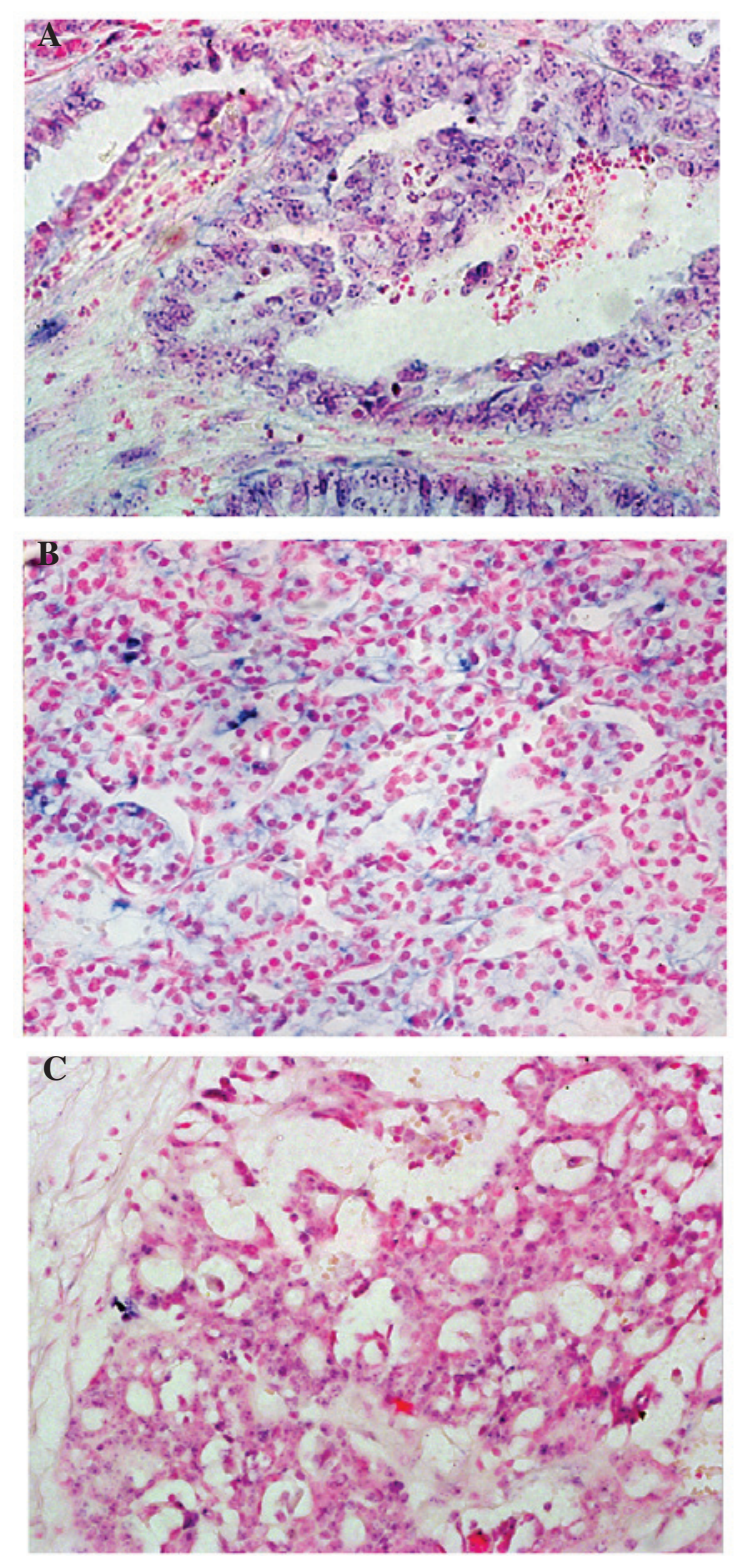

Figure 5. In situ hybridization of miR-510 in LGSC, CCC and HGSC. The signal was visualized by BCIP/NBT solution and the nuclei were counterstained with nuclear fast red. (A) Malignant cells in LGSC exhibited a clear blue signal in the cytoplasm and nucleus. (B) Malignant cells in CCC exhibited a clear blue signal. (C) HGSC exhibited a weak blue signal only. Magnification, x200. HGSC, high-grade serous carcinoma; LGSC, low-grade serous carcinoma; CCC; clear cell carcinoma; miR, microRNA.

and/or no change in the level of miR-510 in HGSC provided further evidence to support the results of the present study and is consistent with the novel model that HGSC belongs to the type II category. In addition, miR-510 may be involved differently in the two broad categories of carcinogenesis.

The function of miR-510 and its validated target genes remain to be fully elucidated. Its oncogenic role has been demonstrated in breast cancer and melanoma $(29,30)$. In melanoma, the miR-506-514 cluster regulates cell growth, apoptosis, invasion and soft agar colony formation, and a sub-cluster of the miR-506-514 phenotype is required for melanocyte transformation (29). In breast cancer, the overexpression of miR-510 increases tumor growth in vivo (30). However, it has also been 
reported that miR-510 may be important as a tumor suppressor. miR-510 is expressed in stage I non-small cell lung cancer, and is deregulated in cases of recurrence (31). Another study in gastric cancer samples demonstrated that lymph node metastases exhibits downregulated expression levels of miR-510, compared with primary cancer samples (32). It appears that miR-510 tends to be highly expressed in localized tumors and may be important in invasion and metastasis.

In conclusion, a unique profile of 83 miRNAs, which were differentially expressed in HGSC and CCC were determined by microarray in the present study, and the majority of these were downregulated at the advanced stage. A total of four miRNAs were validated using RT-qPCR, and miR-510 and miR-129-3p were confirmed to be close associated with the prognosis of patients with EOC. The expression levels of miR-510 in CCC and LGSC were significantly higher than those in HGSC and OSE. These results suggested that miR-510 may have different roles in the two broad categories of carcinogenesis.

\section{Acknowledgements}

This study was supported by the National Basic Research Program of China 973 (program no. 2012CB517600; grant no. 2012CB517603) to Professor Cui Shiying, and was partly supported by a Science Grant to Miss Xiaotang $\mathrm{Yu}$ from the Department of Education, Liaoning Province (grant no. L2011157). The authors would like to thank all family members of the participants for their kind cooperation.

\section{References}

1. Jemal A, Bray F, Center MM, Ferlay J, Ward E and Forman D: Global cancer statistics. CA Cancer J Clin 61: 69-90, 2011.

2. Wang L, Zhu MJ, Ren AM, Wu HF, Han WM, Tan RY and Tu RQ: A ten-microRNA signature identified from a genome-wide microRNA expression profiling in human epithelial ovarian cancer. PLoS One 9: e96472, 2014.

3. Walentowicz P, Sadlecki P, Krintus M, Sypniewska G, Mankowska-Cyl A, Grabiec M and Walentowicz-Sadlecka M: Serum anti-müllerian hormone levels in patients with epithelial ovarian cancer. Int J Endocrinol 2013: 517239, 2013.

4. Siegel R, Ward E, Brawley O and Jemal A: Cancer statistics, 2011: The impact of eliminating socioeconomic and racial disparities on premature cancer deaths. CA Cancer J Clin, 61: 212-236, 2011

5. Kim PS, Djazayeri S and Zeineldin R: Novel nanotechnology approaches to diagnosis and therapy of ovarian cancer. Gynecol Oncol 120: 393-403, 2011.

6. Gilks CB and Prat J: Ovarian carcinoma pathology and genetics: Recent advances. Hum Pathol 40: 1213-1223, 2009.

7. Landen CN Jr, Birrer MJ and Sood AK: Early events in the pathogenesis of epithelial ovarian cancer. J Clin Oncol 26: 995-1005, 2008.

8. Kurman RJ: Origin and molecular pathogenesis of ovarian high-grade serous carcinoma. Ann Oncol 24 (Suppl 10): x16-x21, 2013.

9. Bennett JA, Dong F, Young RH and Oliva E: Clear cell carcinomas of the ovary: Evaluation of prognostic parameters based on a clinicopathologic analysis of 100 cases. Histopathology 66: 808-815, 2015

10. Kinose Y, Sawada K, Nakamura K and Kimura T: The role of microRNAs in ovarian cancer. Biomed Res Int 2014: 249393, 2014.

11. Oom AL, Humphries BA and Yang C: MicroRNAs: Nove Players in cancer diagnosis and therapies. Biomed Res Int 2014: 959461, 2014.

12. Zhang B, Pan X, Cobb GP and Anderson TA: MicroRNAs as oncogenes and tumor suppressors. Dev Biol 302: 1-12, 2007.

13. Ventura A and Jacks T: MicroRNAs and cancer: Short RNAs go a long way. Cell 136: 586-591, 2009.
14. Zhang L, Volinia S, Bonome T, Calin GA, Greshock J, Yang N, Liu CG, Giannakakis A, Alexiou P, Hasegawa K, et al: Genomic and epigenetic alterations deregulate microRNA expression in human epithelial ovarian cancer. Proc Natl Acad Sci USA 105: 7004-7009, 2008.

15. van Jaarsveld MT, Helleman J, Berns EM and Wiemer EA: MicroRNAs in ovarian cancer biology and therapy resistance. Int J Biochem Cell Biol 42: 1282-1290, 2010.

16. Eitan R, Kushnir M, Lithwick-Yanai G, David MB, Hoshen M, Glezerman M, Hod M, Sabah G, Rosenwald S and Levavi H: Tumor microRNA expression patterns associated with resistance to platinum based chemotherapy and survival in ovarian cancer patients. Gynecol Oncol 114: 253-259, 2009.

17. Kandukuri SR and Rao J: FIGO 2013 staging system for ovarian cancer: What is new in comparison to the 1988 staging system? Curr Opin Obstet Gynecol 27: 48-52, 2015.

18. Mavridis K, Stravodimos K and Scorilas A: Downregulation and prognostic performance of microRNA 224 expression in prostate cancer. Clin Chem 59: 261-269, 2013.

19. Zhao JY,Liu CQ,ZhaoHN, Ding YF, Bi T, Wang B, Lin XC, Guo G and Cui SY: Synchronous detection of miRNAs, their targets and downstream proteins in transferred FFPE sections: Applications in clinical and basic research. Methods 58: 156-163, 2012.

20. Yu X, Zhang X, Bi T, Ding Y, Zhao J, Wang C, Jia T, Han D, Guo G, Wang B, et al: MiRNA expression signature for potentially predicting the prognosis of ovarian serous carcinoma. Tumour Biol 34: 3501-3508, 2013.

21. Vilming Elgaaen B, Olstad OK, Haug KB, Brusletto B, Sandvik L, Staff AC, Gautvik KM and Davidson B: Global miRNA expression analysis of serous and clear cell ovarian carcinomas identifies differentially expressed miRNAs including miR-200c-3p as a prognostic marker. BMC Cancer 14: 80, 2014.

22. Lee H, Park CS, Deftereos G, Brusletto B, Sandvik L, Staff AC, Gautvik KM and Davidson B: MicroRNA expression in ovarian carcinoma and its correlation with clinicopathological features. World J Surg Oncol 10: 174, 2012.

23. Banno K, Yanokura M, Iida M, Adachi M, Nakamura K, Nogami Y, Umene K, Masuda K, Kisu I, Nomura H, et al: Application of microRNA in diagnosis and treatment of ovarian cancer. Biomed Res Int 2014: 232817, 2014.

24. Yang D, Sun Y,Hu L, Zheng H, Ji P, Pecot CV, Zhao Y, Reynolds S, Cheng H, Rupaimoole R, et al: Integrated analyses identify a master microRNA regulatory network for the mesenchymal subtype in serous ovarian cancer. Cancer Cell 23: 186-199, 2013.

25. Streicher KL, Zhu W, Lehmann KP, Georgantas RW, Morehouse CA, Brohawn P, Carrasco RA, Xiao Z, Tice DA, Higgs BW, et al: A novel oncogenic role for the miRNA-506-514 cluster in initiating melanocyte transformation and promoting melanoma growth. Oncogene 31: 1558-1570, 2012.

26. Bagnoli M, De Cecco L, Granata A, Nicoletti R, Marchesi E, Alberti P, Valeri B, Libra M, Barbareschi M, Raspagliesi F, et al: Identification of a chrXq27.3 microRNA cluster associated with early relapse in advanced stage ovarian cancer patients. Oncotarget 2: 1265-1278, 2011.

27. Lynch HT, Casey MJ, Snyder CL, Bewtra C, Lynch JF, Butts M and Godwin AK: Hereditary ovarian carcinoma: Heterogeneity, molecular genetics, pathology, and management. Mol Oncol 3: 97-137, 2009.

28. Koshiyama M, Matsumura N and Konishi I: Recent concepts of ovarian carcinogenesis: Type I and type II. Biomed Res Int 2014: 934261, 2014.

29. Streicher KL, Zhu W, Lehmann KP, Georgantas RW Morehouse CA, Brohawn P, Carrasco RA, Xiao Z, Tice DA, Higgs BW, et al: A novel oncogenic role for the miRNA-506-514 cluster in initiating melanocyte transformation and promoting melanoma growth. Oncogene 31: 1558-1570, 2012.

30. Guo QJ, Mills JN, Bandurraga SG, Nogueira LM, Mason NJ, Camp ER, Larue AC, Turner DP and Findlay VJ: MicroRNA-510 promotes cell and tumor growth by targeting peroxiredoxin1 in breast cancer. Breast Cancer Res 15: R70, 2013.

31. Patnaik SK, Kannisto E, Knudsen S and Yendamuri S: Evaluation of microRNA expression profiles that may predict recurrence of localized stage I non-small cell lung cancer after surgical resection. Cancer Res 70: 36-45, 2010.

32. Chen W, Tang Z, Sun Y, Zhang Y, Wang X, Shen Z, Liu F and Qin X: miRNA expression profile in primary gastric cancers and paired lymph node metastases indicates that miR-10a plays a role in metastasis from primary gastric cancer to lymph nodes. Exp Ther Med 3: 351-356, 2012. 